\title{
Schwann Cells Promote Prevascularization and Osteogenesis of Tissue-engineered Bone via Bone Marrow Mesenchymal Stem Cell-derived Endothelial Cells
}

\section{Xinxin Zhang ( $\sim$ xxzhang1983@163.com )}

Department of Orthopaedics, National Cancer Center/National Clinical Research Center for Cancer/Cancer Hospital, Chinese Academy of Medical Sciences and Peking Union Medical College, Beijing, China; https://orcid.org/0000-0001-6202-003X

\section{Xiaorui Jiang}

Qindao University Medical College Affiliated Yantai Yuhuangding Hospital

\section{Shan Jiang}

Southern Medical University First Affiliated Hospital: Southern Medical University Nanfang Hospital

\section{Xiyu Cai}

Fifth Affiliated Hospital of Sun Yat-sen University

\section{Guoxian Pei}

Southern University of Science and Technology Hospital

\section{Research}

Keywords: Schwann cells, endothelial cells, bone marrow-derived mesenchymal stem cells, prevascularization, bone tissue engineering, nestin, TIMP-2

Posted Date: October 14th, 2020

DOI: https://doi.org/10.21203/rs.3.rs-89984/v1

License: (c) (1) This work is licensed under a Creative Commons Attribution 4.0 International License. Read Full License

Version of Record: A version of this preprint was published at Stem Cell Research \& Therapy on July 7th, 2021. See the published version at https://doi.org/10.1186/s13287-021-02433-3. 


\section{Abstract}

Background: Tissue-engineered bone grafts (TEBGs) that undergo vascularization and neurotization evolve into functioning bone tissue. Previously, we verified that implanting sensory nerve tracts into TEBGs promoted osteogenesis. However, the precise mechanisms and interaction between seed cells were not explored. In this study, we hypothesized that neurotization may influence the osteogenesis of TEBGs through vascularization.

Methods: We cultured rat Schwann cells (SCs), aortic endothelial cells (AECs) and bone marrow-derived mesenchymal stem cells (BM-MSCs) and then obtained BM-MSC-derived induced endothelial cells (IECs) and induced osteoblasts (IOBs). IECs and AECs were cultured in SC-conditioned medium (SC-CM) to assess proliferation, migration, capillary-like tube formation and angiogenesis, and the vascular endothelial growth factor (VEGF) levels in the supernatants were detected. We established an indirect coculture model to detect the expression of nestin and VEGF receptors in IECs and tissue inhibitor of metalloproteinase (TIMP)-2 in SCs. Then, SCs, IECs and IOBs were labeled and loaded into a $\beta$-tricalcium phosphate scaffold to induce prevascularization, and the scaffold was implanted into a 6-mm-long defect of rat femurs. Three groups were set up according to the loaded cells: I, SCs and IECs (coculture for 3 days) plus IOBs; II, IECs (culture for 3 days) plus IOBs; III, IOBs. Nestin and TIMP-2 expression and osteogenesis of TEBGs were evaluated at 12 weeks post implantation through histological and radiological assessment.

Results: We found that SC-CM promoted IEC proliferation, migration, capillary-like tube formation and angiogenesis, but no similar effects were observed for AECs. IECs expressed nestin extensively, while AECs barely expressed nestin, and SC-CM promoted the VEGF secretion of IECs. In the coculture model, SCs promoted nestin and VEGF receptor expression in IECs, and IECs inhibited TIMP-2 expression in SCs. The promotion of prevascularized TEBGs by SCs and IECs in group I augmented new bone formation at 6 and 12 weeks. Nestin expression was higher in group I than in the other groups, while TIMP-2 expression was lower at 12 weeks.

Conclusions: This study demonstrated that SCs can promote TEBG osteogenesis via IECs and further revealed the mechanisms related to specific characteristics of IECs, providing preliminary cytological evidence for neurotization of TEBGs.

\section{Background}

Although great progress has been made in the field of bone tissue engineering, large bone defects caused by trauma, infection or tumors remain an unsolved issue. Nerves and blood vessels are both indispensable for normal bone. There is clear evidence that the efficiency of bone ossification is dependent on the level of angiogenesis and vascularization [1]. The vasculature within a graft supplies oxygen and nutrients, removes metabolites and provides specific hormones, growth factors and neurotransmitters to cells seeded in the graft, allowing successful transformation of tissue-engineered 
bone grafts (TEBGs) into functional bone tissues and thereby ensuring their survival and stimulating their activity $[1,2]$. The microscopic architecture of compact tissues of bone is characterized by osteons and Haversian channels containing both nerves and blood supply [3]. The coexistence of interwoven microvasculature and functional nerve fibers in TEB contributes to the formation of physiologically mature bone. The distribution of nerves in bones was recognized very early $[4,5]$. In physiological bone tissue, nerves are distributed throughout the periosteum, cortex and marrow parenchyma and are associated with marrow blood vessels [6]. Nerves, blood vessels and bones interact reciprocally and in a complex manner in tissue-engineered bone (TEB).

It has been confirmed that neuropeptides and neurotrophins secreted by nerve fibers within the musculoskeletal system affect bone [7]. Nerve fibers are able to regulate fundamental biological processes such as remodeling, metabolism, hematopoiesis and angiogenesis in bone tissues [8-10]. Bone is innervated by sensory and sympathetic nerve fibers that, in addition to skeletal pain transmission, play a role in bone metabolism [3]. Many neuropeptides secreted by sensory and autonomic nerves within bone tissues, including substance $P$, calcitonin gene-related peptide from sensory nerves, vasoactive intestinal peptide from parasympathetic nerves, and neuropeptide $Y$ from sympathetic nerves, positively regulate the function of bone cells [11-13]. Similarly, neurotrophins, including nerve growth factor (NGF) and brain-derived neurotrophic factor (BDNF), directly stimulate bone cells $[14,15]$.

Nerves affect blood vessels in the process of TEB construction. Our previous studies concluded that implanting sensory nerve tracts into TEBGs can significantly enhance both vascularization and neurotization simultaneously to obtain a better osteogenic effect [16-18]. Schwann cells (SCs), as glial cells in peripheral nerves, can provide trophic support for axonal regeneration and neurogenesis and secrete a variety of neurotrophic factors, including NGF and BDNF. Previous studies have suggested that NGF and BDNF are able to promote angiogenesis [19-21]. However, some studies have shown that SCderived tissue inhibitor of metalloproteinase (TIMP)-2 inhibits angiogenesis [22, 23]. The interaction between blood vessels and nerves during TEB osteogenesis is complicated.

We hypothesized that neurotization may influence the process of TEBG osteogenesis and that this effect is mediated, at least partly, through vascularization. To explore the interactions between neurotization and vascularization in TEB, it is imperative to understand the relationship between SCs and endothelial cells and the biological mechanisms that underlie the relationship. In this study, we aimed to reveal the relationship between SCs and endothelial cells during TEB construction and provide cytological evidence for simultaneous neurotization and vascularization of TEB.

\section{Methods}

\section{Cell culture and induction}

All animal experiments were approved by the Animal Welfare and Ethics Committee of Southern Medical University. Rat SCs, thoracic aortic endothelial cells (AECs) and bone marrow-derived mesenchymal stem cells (BM-MSCs) were isolated according to previously described protocols [24-27]. BM-MSCs at passage 
3 were differentiated into induced endothelial cells (IECs) or induced osteoblasts (IOBs) by corresponding induction medium for 21 days. The composition of culture medium used were shown in Table 1.

\section{Cell identification}

SCs were characterized by immunocytochemistry staining for S100. A mouse monoclonal antibody against S100 (1:200; Abcam, Cambridge, MA) was used, and the nuclei were counterstained with hematoxylin. AECs were characterized by immunofluorescence staining for von Willebrand factor (vWF). A rabbit polyclonal antibody against vWF (1:400; Abcam) and a DyLight 594-conjugated secondary antibody (1:200; Abcam) were used, and the nuclei were then stained with 4',6-diamidino-2-phenylindole (DAPI; Beyotime, Shanghai, China). Changes in the morphology and organelles of BM-MSCs and IECs were observed by light microscopy and transmission electron microscopy (TEM). IECs were characterized by immunofluorescence staining for VWF and Pecam1. The antibody against vWF (1:400) and a rabbit monoclonal antibody against Pecam1 (1:100; Abcam) were used, and the nuclei were stained with DAPI. IOBs were identified by staining with alkaline phosphatase (ALP; Beyotime) and alizarin red S (SigmaAldrich).

\section{Conditioned medium}

Confluent SCs in 10-cm dishes were rinsed three times with phosphate-buffered saline (PBS), and $10 \mathrm{ml}$ of basal medium (high-glucose DMEM or M199) without FBS was added. This medium was harvested after $48 \mathrm{~h}$ and centrifuged for $5 \mathrm{~min}$ at $3000 \mathrm{xg}$. Then, the supernatant was collected as SC-conditioned medium (CM)-DMEM or SC-CM-M199 and stored at $-70{ }^{\circ} \mathrm{C}$ until use.

\section{Cell proliferation assay}

The proliferation of IECs or AECs in SC-CM-DMEM or SC-CM-M199 supplemented with $2 \%$ FBS (for IECs) or $5 \%$ FBS (for AECs) without other supplements was detected by using the CyQUANT Kit (Thermo Fisher Scientific) according to the manufacturer's instructions. In the control groups, the basal medium was DMEM or M199. The original number of cells in each well of a 96 -well plate was $1.5 \times 10^{3}$ for IECs or $2.5 \times 10^{3}$ for AECs. The fluorescence intensity in triplicate wells each group was measured on days $1,3,5$, 7, 9 and 11 at an excitation wavelength of $485 \mathrm{~nm}$ and an emission wavelength of $530 \mathrm{~nm}$ and converted to cell number according to a standard curve.

\section{Cell migration assay}

An endothelial cell migration assay was performed by using Transwell chambers (pore size of $8 \mu \mathrm{m}, 6.5$ $\mathrm{mm}$ in diameter; Millipore, Billerica, MA). In quadruplicate, IECs or AECs $\left(1.2 \times 10^{4}\right.$ cells/well) were seeded into the upper chambers in $120 \mu$ of DMEM or M199 without FBS or other supplements, and $500 \mu \mathrm{l}$ of SCCM-DMEM or SC-CM-M199 (DMEM or M199 as a control) containing $0.5 \%$ bovine serum albumin (BSA; Beyotime) and $1 \%$ FBS was added to the lower chambers. The upper chambers were removed from the lower chambers after incubation at $37^{\circ} \mathrm{C}$ for six hours and wiped with cotton swabs. The polycarbonate 
membranes were fixed using methanol and stained with crystal violet. The membranes were eluted by using $100 \mu \mathrm{l}$ of $10 \%$ acetic acid. The optical density (OD) of the eluted fluid in triplicate wells was measured at an absorbance of $570 \mathrm{~nm}$.

\section{Capillary-like tube formation assay}

Capillary-like tube formation was detected by using Matrigel (BD Biosciences, San Jose, CA). Liquid Matrigel was placed in a 96-well plate $\left(60 \mu \mathrm{l} /\right.$ well). IECs or AECs $\left(2 \times 10^{4}\right.$ cells/well) were seeded on Matrigel in $40 \mu$ of SC-CM-DMEM or SC-CM-M199. After incubation for $4 \mathrm{~h}$ at $37^{\circ} \mathrm{C}$, phase contrast micrographs were taken. Capillary-like meshes were quantified by ImageJ 1.4.3 software (National Institutes of Health, USA) in random fields in triplicate wells.

\section{Corneal angiogenesis assay}

IECs were resuspended in concentrated SC-CM-DMEM or DMEM at a density of $1 \times 10^{7} \mathrm{cells} / \mathrm{ml}$. A total of $2 \mu \mathrm{l}$ of medium containing cells was homogeneously mixed with $2 \mu \mathrm{l}$ of $1 \%$ gelatin (Aladdin, Shanghai, China). The mixture was injected into the corneas of eight anesthetized eight-week-old female SpragueDawley (SD) rats according to the groups (4 rats per group), which were purchased from the Experimental Center of Southern Medical University (Guangzhou, China). After 12 days, the rats were sacrificed and perfused with waterproof drawing ink (Zhonghua, Shanghai, China) by intracardiac injection. The eyes were fixed with $10 \%$ neutralized buffered formalin, and then the corneas were excised to observe angiogenesis. Vascular segments were quantified by ImageJ 1.4.3 software in random fields in quadruplicate samples.

\section{Indirect coculture of SCs and IECs}

SCs and IECs were indirectly cocultured by using Transwell chambers (pore size of $0.4 \mu \mathrm{m}, 24 \mathrm{~mm}$ in diameter; Millipore). SCs ( $1 \times 10^{4}$ cells/well) were seeded into the upper chambers in $1.5 \mathrm{ml}$ of DMEM supplemented with $10 \%$ FBS without EGM-2 SingleQuots. IECs ( $1 \times 10^{4}$ cells/well) were seeded into the lower chambers in $2.6 \mathrm{ml}$ of the same medium. IECs not cocultured with SCs were plated in the control wells. IECs were collected on days 3 and 7 to examine the effect of SCs on them.

Likewise, IECs $\left(1 \times 10^{4}\right.$ cells/well) were seeded into the upper chambers in $1.5 \mathrm{ml}$ of DMEM supplemented with $10 \%$ FBS without other supplements. SCs $\left(1 \times 10^{4}\right.$ cells/well) were seeded into the lower chambers in $2.6 \mathrm{ml}$ of the same medium. SCs not cocultured with IECs were plated in the control wells. SCs were collected on days 3 and 7 to examine the effect of IECs on them.

\section{Quantitative reverse-transcription polymerase chain reaction (qPCR)}

Total RNA was extracted from IECs, SCs and BM-MSCs by using an RNeasy Mini Kit (Qiagen, Valencia, $\mathrm{CA}$ ) with on-column DNA digestion to eliminate genomic contamination. cDNA was synthesized by using the iScript cDNA Synthesis Kit (Bio-Rad Laboratories, Hercules, CA). Real-time PCR was conducted by 
using SYBR Green PCR Master Mix (Applied Biosystems, Thermo Fisher Scientific). The primer sequences used were shown in Table 2. The fold changes in expression were calculated using the 2- $\Delta \Delta \mathrm{Ct}$ method [28].

\section{Enzyme-linked immunosorbent assay (ELISA)}

Secreted vascular endothelial growth factor (VEGF) levels were detected with a Quantikine® rat VEGF assay (R\&D Systems, Minneapolis, MN) according to the manufacturer's instructions. VEGF concentration in the SC-CM-DMEM was detected firstly. IECs were cultured in SC-CM-DMEM or DMEM with $2 \%$ FBS. The VEGF concentration in the supernatants of the wells was detected by ELISA on days 1, 3, 5, and 7. The medium in all the detected wells was fully replaced $24 \mathrm{~h}$ before each time point at which the supernatant was collected and analyzed.

\section{Western blot (WB) analysis}

Cells were harvested and lysed in radioimmunoprecipitation assay (RIPA) lysis buffer (Beyotime) to extract protein. The supernatants collected from the coculture system or the wells without coculture on days 3 and 7 were concentrated after constant volume, and the protein of supernatants was obtained. Equivalent amounts of protein were separated on a sodium dodecyl sulfate (SDS) gel (Beyotime) and electrotransferred to PVDF membranes (Millipore). WB analysis was performed using the appropriate diluted antibodies. Signals were detected using an ImageQuant LAS System (GE Healthcare Life Sciences, Marlborough, MA). Primary antibodies specific for Flt1, Kdr, nestin (Rat-401) (all from Santa Cruz Biotechnology, Santa Cruz, CA), and TIMP-2 (Thermo Fisher Scientific) and a control antibody specific for $\beta$-actin (Sigma-Aldrich) were used in this experiment.

\section{Immunofluorescence staining}

IECs or AECs were fixed and incubated with a mouse monoclonal antibody against nestin (1:200). Then, they were rinsed and incubated with a secondary antibody conjugated to DyLight 488 (1:200; Abcam). The nuclei were then stained with DAPI.

IECs cultured in SC-CM-DMEM for 7 days were first immunostained for nestin (1:200) as described above and then immunostained for Flt1 (1:200) following the same procedure. Different secondary antibodies, including the DyLight 488-conjugated antibody (1:200) for nestin and a DyLight 405-conjugated antibody (1:200, Abcam) for Flt1, were used. The cells were finally visualized under a TCS-SP5 confocal laser scanning microscope (Leica, Buffalo Grove, IL).

\section{Cell transfection and labeling}

BM-MSCs at passage 3 were infected with lentiviral medium (GeneChem, Shanghai, China) containing enhanced green fluorescent protein (EGFP) or red fluorescent protein (RFP) according to the manufacturer's instructions to obtain BM-MSCs expressing EGFP or RFP, which were called BM-MSCsEGFP or BM-MSCs-RFP, respectively. Stably infected BM-MSCs were selected with $1.5 \mu \mathrm{g} / \mathrm{ml}$ puromycin 
(Solarbio, Beijing, China). BM-MSCs-EGFP were differentiated into IOBs, and BM-MSCs-RFP were differentiated into IECs for scaffold loading. SC nuclei were stained with $100 \mu \mathrm{g} / \mathrm{ml}$ Hoechst 33342 (Beyotime) to obtain labeled SCs.

\section{Preparation of scaffolds}

Sterile cylindrical $\beta$-tricalcium phosphate ( $\beta$-TCP) scaffolds $(6 \mathrm{~mm}$ in length and $4 \mathrm{~mm}$ in diameter; pore size of 200 300 $\mu \mathrm{m}$, porosity volume of $>85 \%$ ) were obtained from Bio-lu Biomaterials Company (Shanghai, China) and presoaked in DMEM overnight. In group I, labeled SCs and IECs-RFP in $200 \mu \mathrm{l}$ of DMEM (1:1 ratio of cell number) were loaded into scaffolds at a concentration of $2 \times 10^{7}$ cells $/ \mathrm{ml}$ using the negative pressure suction method. After $4 \mathrm{~h}$, an additional $10 \mathrm{ml}$ of DMEM was added to each well to completely submerge the scaffolds in medium. The same number of IOBs-EGFP in $200 \mu \mathrm{l}$ of DMEM were loaded into scaffolds that had been incubated for 3 days. After $4 \mathrm{~h}$, the scaffolds were incubated in DMEM for another 3 days. In group II, only IECs-RFP were loaded in the first three days, and other procedures were the same as those in group I. In group III, only IOBs-EGFP were loaded and incubated for 3 days.

\section{Animal models}

Thirty-six ten-week-old male SD rats were purchased from the Experimental Center of Southern Medical University and divided into three groups according to the scaffold groups described above (12 rats per group). The rats were anesthetized and sterilized. The left middle femur was exposed in the intermuscular space through a lateral longitudinal incision. Four holes were drilled in the outer side of the femoral shaft to allow two screws to be placed on either side of the proposed osteotomy site. After periosteal dissection, a 6-mm-long defect was created by using a scroll saw. A scaffold loaded with cells was implanted into the defect. A stainless steel linear reconstruction plate with four holes was then placed along the femur and fixed with four cortical screws. A 20-gauge steel wire was looped between the two screws on either side of the plate to strengthen fixation. The scaffold was fixed to the steel plate with two sutures to keep it stable. The subcutaneous and skin layers were closed in a routine manner.

\section{Radiological observations}

We randomly selected six rats from each group for radiological examination at 6 weeks and 12 weeks. The conditions of X-ray examination included a voltage of $40 \mathrm{kV}$, a current of $50 \mathrm{~mA}$, and an exposure time of $200 \mathrm{msec}$. After the steel plate and screws were removed, micro-CT scans were performed at 12 weeks with an X-ray tube voltage of $80 \mathrm{kV}$, a current of $500 \mu \mathrm{A}$, and an exposure time of $200 \mathrm{msec}$. The average bone volume/total volume (BV/TV \%) values of the femoral defect areas were analyzed for each group.

\section{Histological assessments}


After removing the steel plate and screws at 12 weeks, we obtained femur tissue samples from each group, embedded the samples in paraffin and sectioned scaffold segments. The sections were deparaffinized and blocked, and immunohistochemistry $(\mathrm{IHC})$ staining was performed at 6 weeks with anti-nestin (1:500) and anti-TIMP-2 (1:200) primary antibodies and corresponding secondary antibodies. $\mathrm{H} \& \mathrm{E}$ and Masson staining were performed at postoperative 12 weeks according to routine procedures.

\section{Statistical analysis}

The data obtained are presented as the means \pm SDs. The data from the cell and animal model experiments were analyzed using Student's t-test (parametric data) or the Mann-Whitney test (nonparametric data). GraphPad Prism 8.0.1 software (GraphPad Software Inc., San Diego, CA) was used to perform calculations and generate graphs. $P<0.05$ was considered statistically significant $(* \star \star P<0.001$, **P $<0.01$ and $* P<0.05$; ns=nonsignificant).

\section{Results}

\section{Identification of cells}

To identify SCs, AECs, BM-MSCs, IECs and IOBs, we performed immunocytochemistry or immunofluorescence staining and other specific staining. The cytoplasm of SCs was positively stained for S100 (Fig. 1A, B). The cytomembrane and cytoplasm of AECs were positively stained for vWF (Fig. 1C, D). The morphology of the IECs changed from the fibrous spindle appearance of BM-MSCs to a cobblestone-like morphology and were positively stained for VWF and Pecam1 (Fig. 1E-H). The qPCR results showed that the expression levels of $C D 34$, kinase insert domain receptor (Kdr), nitric oxide synthase (Nos)2 and Nos3 in IECs were significantly increased compared with those in BM-MSCs (Fig. 1I). The TEM results showed that IECs had more synapses, organelles and phagocytic vesicles than BMMSCs and that Weibel-Palade (W-P) bodies were observed near the nuclei of IECs (Fig. 1J, K). BM-MSCderived IOBs showed positive alizarin ALP and red S staining (Fig. 1L, M).

\section{SC-CM promotes IEC proliferation, migration, capillary-like tube formation and angiogenesis}

The number of IECs cultured in SC-CM-DMEM was significantly higher than that in the control group at each time point. The number of AECs cultured in SC-CM-M199 was significantly lower that of the control group at 1 day and no significant difference was showed after 3 days (Fig. 2A). There were more migrated IECs in the SC-CM-DMEM wells than in the DMEM wells, while relatively less migrated AECs in the SC-CM-M199 wells than in the M199 wells (Fig. 2B). The OD values of the eluted fluid illustrated the same result (Fig. 2C). More capillary-like tube structures were found in the CM wells than in the control wells for IECs, while no significant difference was shown for AECs (Fig. 2D), as shown by capillary-like tube structure counting (Fig. 2E). More vessels and vascular segment counting were observed in the corneas of the SC-CM-DMEM group than in the corneas of the control group on the 12th day (Fig. 2F, G).

\section{SCs and IECs interact in a coculture system}


First, the VEGF concentration in SC-CM-DMEM was detected, and the detected value $(499.85 \mathrm{pg} / \mathrm{ml}$ in SCCM-DMEM) confirmed that SCs secreted VEGF. The VEGF concentration in the supernatant during the coculture process was also detected, and the results showed that the VEGF concentration secreted only by IECs in the SC-CM wells increased successively at each time point and was higher significantly than the control group at each time point (Fig. 3A).

We compared nestin expression in IECs and AECs, and the results showed that IECs expressed nestin extensively, while AECs barely expressed nestin (Fig. 3B). Double immunofluorescence staining showed that nestin and Flt1 were simultaneously expressed more strongly in IECs in SC-CM-DMEM wells than in the DMEM wells (Fig. 3C). To further investigate the interaction between SCs and IECs, we detected nestin and VEGF receptor expression levels in IECs and TIMP-2 expression levels in SCs in the coculture system. The WB results showed that the expression levels of nestin, Flt1 and Kdr were significantly higher in IECs cocultured with SCs than in IECs cultured without SCs and revealed an increasing trend as the time in coculture increased (Fig. 3D). The qPCR results showed that the expression level of TIMP-2 in SCs cocultured with IECs was significantly lower than the control wells at days 3 and 7, while matrix metalloproteinase (MMP)-14 expression was significantly higher during the process of coculture (Fig. 3E). The results of secreted TIMP-2 expression in the supernatants showed an decreasing trend when SCs were cocultured with IECs, and the secreted TIMP-2 expression level was significantly lower in the supernatants of the SC and IEC coculture system than without coculture (Fig. 3F).

\section{Implantation of scaffolds with labeled cells}

To observe the attachment and growth of cells loaded on scaffolds, we labeled BM-MSCs with EGFP and RFP and induced the labeled BM-MSCs to differentiate into IOBs-EGFP and IECs-RFP (Fig. 4A). The nuclei of SCs were labeled with Hoechst 33342 (Fig. 4B). Scanning electron microscopy (SEM) revealed that the seeded cells adhered well to the scaffolds (Fig. 4C). The labeled SCs, IOBs-EGFP and IECs-RFP grew well on the scaffolds, as observed under a fluorescent inverted microscope (Fig. 4D). The cell-loaded scaffolds were successfully implanted into the femoral defects of rats, and the internal fixations were placed firmly (Fig. 4E-H). The procedures and groups of the experimental design are displayed in Fig. 4 I.

\section{Radiological assessment}

At 6 weeks, the X-ray results showed that the bone defects of the rats in each group had not completely healed after the operation. In group I, the TEBG material was absorbed, the internal density was uneven, and a small number of bony calluses formed on the surface of the TEBG. In groups II and III, the density of the TEBG material was significantly different from the density of the surrounding bone, the fracture line was still clearly visible, and there was no bony callus formation. Twelve weeks after the operation, $X$ ray imaging showed that the bone defects of the rats in group I had healed and that the fracture line had disappeared. Bony calluses were evenly distributed at the defect site and bridged the fracture ends. The bone defects of rats in group II had partially healed, and the fracture line was obscure and showed some bony callus formation. In group III, the bone defect had not healed, and the fracture line was still clear without visible bony callus formation (Fig. 5A). 
Micro-CT at postoperative 12 weeks more intuitively showed the difference in TEBG osteogenesis in each group in three dimensions and coronal images (Fig. 5B). The BV/TV \% values of the rats in group I were significantly higher than those of the other two groups, and group III showed the lowest value of the three groups (Fig. 5C).

\section{Histological assessment}

For IHC staining, in group I, the nestin high expression areas were significantly more than the other two groups (Fig. 6A, B), while TIMP-2 expression areas were significantly less (Fig. 6G, H). Group II showed sporadic and individual nestin expression cells (Fig. 6C, D) and relatively more TIMP-2 expression areas (Fig. $6 \mathrm{I}, \mathrm{J})$. In the areas of bony collagen formation and growth in group III, almost no nestin expression was observed (Fig. 6E, F), but obvious and more TIMP-2 expression was visible (Fig. 6K, L).

At 12 weeks, in group I, most of the scaffolds degraded, and regenerated woven bone formed in the implanted areas. Thickened trabecular bones grew in some areas where Masson staining showed obvious bone growth (Fig. 6M, P). Group II showed that some of the scaffolds degraded and collagen fibrous tissue grew inside the scaffolds, where a small amount of new bone could be observed through H\&E and Masson staining (Fig. 6N, Q). Group III showed similar histological changes to group II, while fewer scaffolds were absorbed and new bone growth was observed (Fig. 60, R).

In summary, the results of this study further revealed the mechanism of interactions between SCs and endothelial cells in the process of construction of TEB. SCs promoted the angiogenesis- and vascularization-related activities of BM-MSC derived IECs by regulating nestin and TIMP-2, in which MMP-14 and VEGF and VEGF receptors involved, whereas IECs inhibited TIMP-2 secretion of SCs by autologous nestin expression, eventually efficiently induced prevascularization and osteogenesis of TEBG (Fig. 7).

\section{Discussion}

The bone is fully vascularized and innervated where blood vessels and nerve fibers closely interact [29]. Blood vessels and nerve fibers, which exist throughout the entire bone, including the periosteum, bone marrow, and mineralized parts of the bone, are involved in bone formation and development $[29,30]$. Vascular and neural involvement is the basis for the construction of normal functional bone tissue during TEBG osteogenesis. To date, numerous studies have focused on the vascularization of TEB, but far less is known regarding the neurotization of TEB. Therefore, our group proposed constructing TEB that is simultaneously highly vascularized and neurotized [31]. We made some efforts to construct neurovascularized TEB, including implanting sensory nerve tracts through microsurgical techniques, which is a feasible method for constructing neurotized TEB [16-18, 31]. In this study, we revealed a correlation between neural and vascular seed cells in the construction of TEBs and verified that SCs and IECs promote the osteogenesis of prevascularized TEBGs. 
BM-MSCs can be induced to differentiate into endothelial cells by some cell factors, including VEGF and basic fibroblast growth factor (bFGF) [32,33]. In this study, we used BM-MSCs as an alternative source of IECs by using EGM-2 SingleQuots, which includes VEGF, bFGF, insulin-like growth factor (IGF) and epidermal growth factor (EGF). The results of cell identification showed that the morphology of IECs, including the appearance of the cells and their organelles, was changed and that the expression of the endothelial cell-specific genes vWF and Pecam1 and Nos2 and Nos3, which are genes related to endothelial cell function, was significantly increased in IECs compared to BM-MSCs. IECs formed capillary-like tube structures and underwent angiogenesis in subsequent experiments. These findings confirm that IECs can be considered sources of vasculature with effective biochemical functions for bone tissue engineering.

As the component cells of the myelin sheath, SCs are commonly used as seed cells in neural tissue engineering [34]. SCs not only improve axonal regeneration but also secrete VEGF to play a protective role in axonal growth [35]. In addition, sensory nerves or SCs can induce arterial marker expression in embryonic endothelial cells by locally secreting VEGF [36]. SCs have also been shown to promote human umbilical vein cell (HUVEC) migration through VEGF [37]. Transplantation of a combination of microencapsulated SCs and BM-MSCs has been verified to augment angiogenesis by increasing local VEGF levels [38]. In the present study, we found that SC-CM promoted IEC proliferation, migration, capillary-like tube formation and angiogenesis. As shown by ELISA, VEGF was present in SC-CM at a relatively high concentration. The VEGF concentration also gradually increased with time in SC and IEC cocultures. VEGF is well known to be a key molecule for the proliferation, migration and angiogenesis of endothelial cells [39]. Thus, VEGF was speculated to be an important factor through which SC-CM promoted IEC proliferation, migration and angiogenesis in this study.

However, some previous studies have confirmed that SC-CM inhibits AEC angiogenesis in vitro and in vivo through TIMP-2 $[22,23]$. We examined the effect of SC-CM on AECs and found that SC-CM did not significantly promote AEC proliferation, migration or capillary-like tube formation. We further found that IECs expressed nestin extensively, while AECs barely expressed nestin. Nestin has been characterized as a potent marker of angiogenesis and neovascularization because it is expressed in endothelial cells with stem-like characteristics but not in mature endothelial cells [40]. Moreover, it has been confirmed that nestin expression is induced in capillary endothelial cells through the VEGF signaling pathway [41]. VEGF facilitates nestin expression to induce endothelial cell migration and angiogenesis by acting on VEGF receptors, including Flt1 $[42,43]$. The results of qPCR and immunofluorescence staining in our study also showed a significant increase in nestin and Flt1 expression in IECs upon stimulation with SCs. Therefore, the difference in the effect of SCs on endothelial cells is due, at least in part, to the differential expression of nestin in endothelial cells of different origins. It can be inferred that SC-derived VEGF can promote IEC proliferation, migration and angiogenesis in IECs through nestin.

Angiogenesis is thought to be regulated by the balance between inducers and inhibitors within a given microenvironment [44]. As a member of the TIMP family, TIMP-2 has been shown to suppress angiogenesis to inhibit the activities of MMPs $[23,45]$. In a noncontact coculture system, SCs affect IECs 
by secreting exogenous molecules, and IECs also affect SCs. Our results showed that TIMP-2 expression was inhibited in SCs cocultured with IECs and that this inhibition persisted throughout the duration of coculture. To compare the effects of endothelial cells of different origins on SCs, we also analyzed SCs cocultured with AECs. However, TIMP-2 was not observed to have an obvious inhibitory effect. MSCs have been confirmed to inhibit TIMP-2 generation and support MMP release by cocultured cardiac fibroblasts or skeletal muscle cells $[46,47]$. In our experiment, qPCR showed that MMP-14 expression was slightly enhanced in SCs cocultured with IECs. TIMP-2 is known to extensively inhibit angiogenesis by binding to MMP-14 and other MMPs [45]. Thus, we speculated that IECs retain some of the features and functions of BM-MSCs, allowing them to inhibit the secretion and effect of exogenous TIMP-2 to subsequently enhance angiogenesis and vascularization.

We used a large segmental femoral defect model in rats in this study. Critical-sized defects (CSDs) are commonly 1.5 to 2 times the diameter of long tubular bones [48]. We created a $6-\mathrm{mm}$-long femoral defect in ten-week-old male SD rats, which had a middle femur cross-section diameter of 25 to $30 \mathrm{~mm}$. Tenweek-old SD rats have a relatively low growth rate of long bones, which allowed the longitudinal stability of the femur and the consistency of the long bone defect during the observation period.

Prevascularization involves vascularization of the scaffold in vitro or in vivo and is a promising method for enhancing the vascularization of tissue constructs upon implantation [49]. We previously performed prevascularization with the insertion of vascular bundle into scaffolds, which can promote the recruitment of endogenous cells and collagen deposition to establish fast-acting angiogenesis and osteogenesis $[50,51]$. Prevascularization also induced by endothelial cells in vitro, which can form original vessel structures in vitro to achieve better vascularization and osteogenic efficacy, has been used in TEB construction before implantation $[52,53]$. Our experiments also adopted this method; IECs and SCs were preseeded and cultured before IOBs were seeded and implanted into femoral defects in rats. SCs have dual angiogenic and neurotrophic effects during prevascularization and TEB construction. Moreover, SCs have been shown to enhance osteoblast proliferation and differentiation [54]. Thus, SCs probably have three effects, namely, promoting vascularization, underlying neurotization, and promoting osteogenesis, in TEB construction. In the present study, we observed the effects of SCs on vascularization and osteogenesis in a rat model through radiological and histological assessments and verified that nestin expression increased and TIMP-2 expression decreased due to the involvement of SCs in vivo. It is true that the relationship between vascularization, neurotization and osteogenesis is complex due to the number of factors involved. Indeed, SCs are only a basic factor required for the neurotization of TEB; further investigation of other factors associated with nerves, including sensory neurons, which are related to osteoblasts and SCs, and related mechanisms underlying TEB are urgently needed [55].

\section{Conclusions}

A variety of cells are involved in the process of osteogenesis and functionalization of TEBGs. Exploring the interaction between seed cells is important to find ways to promote bone regeneration. In this study, we revealed that SCs can promote the proliferation, migration and angiogenesis of BM-MSC-derived IECs 
by regulating nestin and autologous TIMP-2. Consequently, SCs promote TEBG vascularization and osteogenesis to repair rat femoral large segmental defects. Meanwhile, our results provide preliminary cytological evidence to support the neurotization of TEB.

\section{List Of Abbreviations}




\begin{tabular}{|c|c|}
\hline AECs & aortic endothelial cells \\
\hline ALP & alkaline phosphatase \\
\hline Ara-C & cytosine-B-arabinoside hydrochloride \\
\hline BDNF & brain-derived neurotrophic factor \\
\hline bFGF & basic fibroblast growth factor \\
\hline BM-MSCs & bone marrow-derived mesenchymal stem cells \\
\hline BSA & bovine serum albumin \\
\hline BV/TV & bone volume/total volume \\
\hline$\beta$-ТСР & $\beta$-tricalcium phosphate \\
\hline CSDs & critical-sized defects \\
\hline $\mathrm{CM}$ & conditioned medium \\
\hline DAPI & 4',6-diamidino-2-phenylindole \\
\hline DMEM & Dulbecco's modified Eagle's medium \\
\hline ECGS & Endothelial Cell Growth Supplement \\
\hline EGF & epidermal growth factor \\
\hline EGFP & enhanced green fluorescent protein \\
\hline EGM-2 SingleQuots & Endothelial Growth Medium-2 supplements and factors \\
\hline ELISA & enzyme-linked immunosorbent assay \\
\hline FBS & fetal bovine serum \\
\hline GAPDH & glyceraldehyde-3-phosphate dehydrogenase \\
\hline HUVEC & human umbilical vein cell \\
\hline IECs & induced endothelial cells \\
\hline IGF & insulin-like growth factor \\
\hline $\mathrm{IHC}$ & immunohistochemistry \\
\hline IOBs & induced osteoblasts \\
\hline Kdr & kinase insert domain receptor \\
\hline M199 & medium 199 \\
\hline MMP & matrix metalloproteinase \\
\hline NGF & nerve growth factor \\
\hline
\end{tabular}




\begin{tabular}{|ll|}
\hline Nos & nitric oxide synthase \\
\hline PBS & optical density \\
\hline qPCR & phosphate-buffered saline \\
\hline RFP & quantitative reverse-transcription polymerase chain reaction \\
\hline RIPA & red fluorescent protein \\
\hline SCs & radioimmunoprecipitation assay \\
\hline SD & Schwann cells \\
\hline SDS & Sprague-Dawley \\
\hline SEM & sodium dodecyl sulfate \\
\hline TEB & scanning electron microscopy \\
\hline TEBGs & tissue-engineered bone \\
\hline TEM & tissue-engineered bone grafts \\
\hline TIMP-2 & transmission electron microscopy \\
\hline VEGF & tissue inhibitor of metalloproteinase-2 \\
\hline VWF & vascular endothelial growth factor \\
\hline WB & von Willebrand factor \\
\hline
\end{tabular}

\section{Declarations}

\section{Ethics approval and consent to participate}

All procedures on rats were performed in the authorized animal care facility and were approved by the Animal Welfare and Ethics Committee of Southern Medical University. All methods were performed in accordance with the relevant guidelines and regulations of the People's Republic of China.

\section{Consent for publication}

Not applicable.

\section{Availability of data and materials}

All supporting data and materials are included in the article.

\section{Competing interests}


The authors declare that they have no competing interests.

\section{Funding}

This study was supported by the PUMC Fund of the Funds for the Central Universities (no. 3332018077), Shandong Provincial Natural Science Foundation Project (no. ZR2019MH073), Guangdong Provincial Natural Science Foundation Project (no. 2018A030313647), and Beijing Hope Run Special Fund of Cancer Foundation of China (no. LC2016B09).

\section{Authors' contributions}

$\mathrm{XZ}$ and $\mathrm{XJ}$ designed and performed the research, analyzed the data and wrote the paper. SJ and XC supplied technical assistance on establishing the rat bone defect models. GP conceived of the study, reviewed the data, revised and approved the final version of the manuscript. All authors read and approved the final manuscript.

\section{Acknowledgements}

We thank American Journal Experts (AJE) for their help in editing the manuscript.

\section{References}

1. Filipowska J, Tomaszewski KA, Niedzwiedzki L, Walocha JA, Niedzwiedzki T. The role of vasculature in bone development, regeneration and proper systemic functioning. Angiogenesis. 2017;20:291-302.

2. Brandi ML, Collin-Osdoby P. Vascular biology and the skeleton. J Bone Miner Res. 2006;21:183-92.

3. Pirosa A, Gottardi R, Alexander PG, Tuan RS. Engineering in-vitro stem cell-based vascularized bone models for drug screening and predictive toxicology. Stem Cell Res Ther. 2018;9:112.

4. Cooper RR. Nerves in cortical bone. Science. 1968;160:327-8.

5. Thurston TJ. Distribution of nerves in long bones as shown by silver impregnation. J Anat. 1982;134:719-28.

6. McCredie J. Nerves in bone: the silent partners. Skeletal Radiol. 2007;36:473-5.

7. Garcia-Castellano JM, Diaz-Herrera P, Morcuende JA. Is bone a target-tissue for the nervous system? New advances on the understanding of their interactions. lowa Orthop J. 2000;20:49-58.

8. Elefteriou F. Impact of the Autonomic Nervous System on the Skeleton. Physiol Rev. 2018;98:1083112.

9. Hanoun M, Maryanovich M, Arnal-Estape A, Frenette PS. Neural regulation of hematopoiesis, inflammation, and cancer. Neuron. 2015;86:360-73.

10. Elefteriou F, Campbell P, Ma Y. Control of bone remodeling by the peripheral sympathetic nervous system. Calcif Tissue Int. 2014;94:140-51. 
11. Tuzmen C, Campbell PG. Crosstalk between neuropeptides SP and CGRP in regulation of BMP2induced bone differentiation. Connect Tissue Res. 2018;59:81-90.

12. Yoo YM, Kwag JH, Kim KH, Kim CH. Effects of neuropeptides and mechanical loading on bone cell resorption in vitro. Int J Mol Sci. 2014;15:5874-83.

13. Liu S, Jin D, Wu JQ, Xu ZY, Fu S, Mei G, Zou ZL, Ma SH. Neuropeptide Y stimulates osteoblastic differentiation and VEGF expression of bone marrow mesenchymal stem cells related to canonical Wnt signaling activating in vitro. Neuropeptides. 2016;56:105-13.

14. Liu Q, Lei L, Yu T, Jiang T, Kang Y. Effect of Brain-Derived Neurotrophic Factor on the Neurogenesis and Osteogenesis in Bone Engineering. Tissue Eng Part A. 2018;24:1283-92.

15. Yada M, Yamaguchi K, Tsuji T. NGF stimulates differentiation of osteoblastic MC3T3-E1 cells. Biochem Biophys Res Commun. 1994;205:1187-93.

16. Fan JJ, Mu TW, Qin JJ, Bi L, Pei GX. Different effects of implanting sensory nerve or blood vessel on the vascularization, neurotization, and osteogenesis of tissue-engineered bone in vivo. Biomed Res Int. 2014;2014:412570.

17. Chen SY, Qin JJ, Wang L, Mu TW, Jin D, Jiang S, Zhao PR, Pei GX. Different effects of implanting vascular bundles and sensory nerve tracts on the expression of neuropeptide receptors in tissueengineered bone in vivo. Biomed Mater. 2010;5:55002.

18. Wu Y, Jing D, Ouyang H, Li L, Zhai M, Li Y, Bi L, Guoxian P. Pre-implanted Sensory Nerve Could Enhance the Neurotization in Tissue-Engineered Bone Graft. Tissue Eng Part A. 2015;21:2241-9.

19. Lazarovici P, Gazit A, Staniszewska I, Marcinkiewicz C, Lelkes PI. Nerve growth factor (NGF) promotes angiogenesis in the quail chorioallantoic membrane. Endothelium. 2006;13:51-9.

20. Lazarovici P, Marcinkiewicz C, Lelkes PI. Cross talk between the cardiovascular and nervous systems: neurotrophic effects of vascular endothelial growth factor (VEGF) and angiogenic effects of nerve growth factor (NGF)-implications in drug development. Curr Pharm Des. 2006;12:2609-22.

21. Sun C, Hu Y, Chu Z, Huang J, Zhang L. The effect of brain-derived neurotrophic factor on angiogenesis. J Huazhong Univ Sci Technolog Med Sci. 2009;29:139-43.

22. Huang D, Rutkowski JL, Brodeur GM, Chou PM, Kwiatkowski JL, Babbo A, Cohn SL. Schwann cellconditioned medium inhibits angiogenesis in vitro and in vivo. Med Pediatr Oncol. 2000;35:590-2.

23. Huang D, Rutkowski JL, Brodeur GM, Chou PM, Kwiatkowski JL, Babbo A, Cohn SL. Schwann cellconditioned medium inhibits angiogenesis. Cancer Res. 2000;60:5966-71.

24. Tao Y. Isolation and culture of Schwann cells. Methods Mol Biol. 2013;1018:93-104.

25. Weinstein DE, Wu R. Isolation and purification of primary Schwann cells. Curr Protoc Neurosci. 2001;Chapter 3:3-17.

26. Mohamed HE, Elswefy SE, Rashed LA, Younis NN, Shaheen MA, Ghanim AM. Bone marrow-derived mesenchymal stem cells effectively regenerate fibrotic liver in bile duct ligation rat model. Exp Biol Med (Maywood). 2016;241:581-91. 
27. Magid R, Martinson D, Hwang J, Jo H, Galis ZS. Optimization of isolation and functional characterization of primary murine aortic endothelial cells. Endothelium. 2003;10:103-9.

28. Livak KJ, Schmittgen TD. Analysis of relative gene expression data using real-time quantitative PCR and the 2(-Delta Delta C(T)) Method. Methods. 2001;25:402-8.

29. Marrella A, Lee TY, Lee DH, Karuthedom S, Syla D, Chawla A, Khademhosseini A, Jang HL. Engineering vascularized and innervated bone biomaterials for improved skeletal tissue regeneration. Mater Today (Kidlington). 2018;21:362-76.

30. Grassel SG. The role of peripheral nerve fibers and their neurotransmitters in cartilage and bone physiology and pathophysiology. Arthritis Res Ther. 2014;16:485.

31. Fan J, Bi L, Jin D, Wei K, Chen B, Zhang Z, Pei G. Microsurgical techniques used to construct the vascularized and neurotized tissue engineered bone. Biomed Res Int. 2014;2014:281872.

32. Yue WM, Liu W, Bi YW, He XP, Sun WY, Pang XY, Gu XH, Wang XP. Mesenchymal stem cells differentiate into an endothelial phenotype, reduce neointimal formation, and enhance endothelial function in a rat vein grafting model. Stem Cells Dev. 2008;17:785-93.

33. Oswald J, Boxberger S, Jorgensen B, Feldmann S, Ehninger G, Bornhauser M, Werner C. Mesenchymal stem cells can be differentiated into endothelial cells in vitro. Stem Cells. 2004;22:37784.

34. Zhang PX, Han N, Kou YH, Zhu QT, Liu XL, Quan DP, Chen JG, Jiang BG. Tissue engineering for the repair of peripheral nerve injury. Neural Regen Res. 2019;14:51-8.

35. Taiana MM, Lombardi R, Porretta-Serapiglia C, Ciusani E, Oggioni N, Sassone J, Bianchi R, Lauria G. Neutralization of schwann cell-secreted VEGF is protective to in vitro and in vivo experimental diabetic neuropathy. PLoS One. 2014;9:e108403.

36. Mukouyama YS, Shin D, Britsch S, Taniguchi M, Anderson DJ. Sensory nerves determine the pattern of arterial differentiation and blood vessel branching in the skin. Cell. 2002;109:693-705.

37. Ramos T, Ahmed M, Wieringa P, Moroni L. Schwann cells promote endothelial cell migration. Cell Adh Migr. 2015;9:441-51.

38. Wang Y, Zhang G, Hou Y, Chen J, Wang J, Zou C, Li D, Li H, Zhang Q, Wang A, Fan Q. Transplantation of microencapsulated Schwann cells and mesenchymal stem cells augment angiogenesis and improve heart function. Mol Cell Biochem. 2012;366:139-47.

39. Melincovici CS, Bosca AB, Susman S, Marginean M, Mihu C, Istrate M, Moldovan IM, Roman AL, Mihu $\mathrm{CM}$. Vascular endothelial growth factor (VEGF) - key factor in normal and pathological angiogenesis. Rom J Morphol Embryol. 2018;59:455-67.

40. Suzuki S, Namiki J, Shibata S, Mastuzaki Y, Okano H. The neural stem/progenitor cell marker nestin is expressed in proliferative endothelial cells, but not in mature vasculature. J Histochem Cytochem. 2010;58:721-30.

41. Takahashi N, Itoh MT, Ishizuka B. Human chorionic gonadotropin induces nestin expression in endothelial cells of the ovary via vascular endothelial growth factor signaling. Endocrinology. 2008;149:253-60. 
42. Liang ZW, Wang Z, Chen H, Li C, Zhou T, Yang Z, Yang X, Yang Y, Gao G, Cai W. Nestin-mediated cytoskeletal remodeling in endothelial cells: novel mechanistic insight into VEGF-induced cell migration in angiogenesis. Am J Physiol Cell Physiol. 2015;308:C349-58.

43. Krum JM, Rosenstein JM. Transient coexpression of nestin, GFAP, and vascular endothelial growth factor in mature reactive astroglia following neural grafting or brain wounds. Exp Neurol. 1999;160:348-60.

44. Pratheeshkumar $P$, Kuttan G. Vernolide-A inhibits tumour specific angiogenesis by regulating proinflammatory cytokines, VEGF, MMPs and TIMP. Eur J Pharmacol. 2011;656:10-8.

45. McLaughlin SL, Ice RJ, Rajulapati A, Kozyulina PY, Livengood RH, Kozyreva VK, Loskutov YV, Culp MV, Weed SA, Ivanov AV, Pugacheva EN. NEDD9 depletion leads to MMP14 inactivation by TIMP2 and prevents invasion and metastasis. Mol Cancer Res. 2014;12:69-81.

46. Mias C, Lairez O, Trouche E, Roncalli J, Calise D, Seguelas MH, Ordener C, Piercecchi-Marti MD, Auge N, Salvayre AN, Bourin P, Parini A, Cussac D. Mesenchymal stem cells promote matrix metalloproteinase secretion by cardiac fibroblasts and reduce cardiac ventricular fibrosis after myocardial infarction. Stem Cells. 2009;27:2734-43.

47. Sassoli C, Nosi D, Tani A, Chellini F, Mazzanti B, Quercioli F, Zecchi-Orlandini S, Formigli L. Defining the role of mesenchymal stromal cells on the regulation of matrix metalloproteinases in skeletal muscle cells. Exp Cell Res. 2014;323:297-313.

48. Cook SD, Wolfe MW, Salkeld SL, Rueger DC. Effect of recombinant human osteogenic protein-1 on healing of segmental defects in non-human primates. J Bone Joint Surg Am. 1995;77:734-50.

49. Lin Y, Huang S, Zou R, Gao X, Ruan J, Weir MD, Reynolds MA, Qin W, Chang X, Fu H, Xu H. Calcium phosphate cement scaffold with stem cell co-culture and prevascularization for dental and craniofacial bone tissue engineering. Dent Mater. 2019;35:1031-41.

50. Wang L, Fan H, Zhang ZY, Lou AJ, Pei GX, Jiang S, Mu TW, Qin JJ, Chen SY, Jin D. Osteogenesis and angiogenesis of tissue-engineered bone constructed by prevascularized $\beta$-tricalcium phosphate scaffold and mesenchymal stem cells. Biomaterials. 2010;31:9452-61.

51. Cheng P, Li D, Gao Y, Cao T, Jiang H, Wang J, Li J, Zhang S, Song Y, Liu B, Wang C, Yang L, Pei G. Prevascularization promotes endogenous cell-mediated angiogenesis by upregulating the expression of fibrinogen and connective tissue growth factor in tissue-engineered bone grafts. Stem Cell Res Ther. 2018;9:176.

52. Yu H, VandeVord PJ, Mao L, Matthew HW, Wooley PH, Yang SY. Improved tissue-engineered bone regeneration by endothelial cell mediated vascularization. Biomaterials. 2009;30:508-17.

53. Cipriano J, Lakshmikanthan A, Buckley C, Mai L, Patel H, Pellegrini M, Freeman JW. Characterization of a prevascularized biomimetic tissue engineered scaffold for bone regeneration. J Biomed Mater Res B Appl Biomater. 2019.

54. Cai XX, Luo E, Yuan Q. Interaction between Schwann cells and osteoblasts in vitro. Int J Oral Sci. 2010;2:74-81. 
55. Haastert K, Semmler N, Wesemann M, Rucker M, Gellrich NC, Grothe C. Establishment of cocultures of osteoblasts, Schwann cells, and neurons towards a tissue-engineered approach for orofacial reconstruction. Cell Transplant. 2006;15:733-44.

\section{Tables}

Table 1 Composition of cell culture medium

\begin{tabular}{|c|c|c|}
\hline Culture medium & Composition & Manufacturer's name and location \\
\hline $\mathrm{SC}$ regular medium & $\begin{array}{l}\text { DMEM containing: } \\
10 \% \text { FBS } \\
2 \mu \mathrm{M} \text { forskolin } \\
10 \mathrm{ng} / \mathrm{ml} \text { heregulin- } \beta-1\end{array}$ & $\begin{array}{l}\text { HyClone, Thermo Fisher Scientific, Pittsburgh, } \\
\text { PA Gibco, USA } \\
\text { Gibco, Gaithersburg, MD } \\
\text { BioVision, Milpitas, CA } \\
\text { PeproTech, Rocky Hill, NJ }\end{array}$ \\
\hline $\begin{array}{l}\text { SC medium for } \\
\text { purification }\end{array}$ & $\begin{array}{l}\text { DMEM containing: } \\
\text { 10\% FBS } \\
10 \mu \mathrm{M} \text { Ara-C }\end{array}$ & $\begin{array}{l}\text { Hyclone } \\
\text { Gibco } \\
\text { Sigma-Aldrich, St. Louis, MO }\end{array}$ \\
\hline AEC medium & $\begin{array}{l}\text { M199 containing: } \\
5 \% \text { FBS } \\
20 \mu \mathrm{g} / \mathrm{ml} \text { ECGS } \\
100 \mu \mathrm{g} / \mathrm{ml} \text { heparin }\end{array}$ & $\begin{array}{l}\text { Hyclone } \\
\text { Gibco } \\
\text { ScienCell, Carlsbad, CA } \\
\text { Sigma-Aldrich }\end{array}$ \\
\hline BM-MSC medium & $\begin{array}{l}\text { DMEM containing: } \\
\text { 10\% FBS }\end{array}$ & $\begin{array}{l}\text { Hyclone } \\
\text { Gibco }\end{array}$ \\
\hline $\begin{array}{l}\text { IEC induction } \\
\text { medium }\end{array}$ & $\begin{array}{l}\text { DMEM ( } 4.5 \mathrm{~g} / \mathrm{l} \text { glucose) } \\
\text { containing: } \\
\text { EGM-2 SingleQuots }\end{array}$ & $\begin{array}{l}\text { Hyclone } \\
\text { Lonza, Basel, Switzerland }\end{array}$ \\
\hline $\begin{array}{l}\text { IOB induction } \\
\text { medium }\end{array}$ & $\begin{array}{l}\text { DMEM ( } 4.5 \mathrm{~g} / \mathrm{l} \text { glucose }) \\
\text { containing: } \\
10 \mathrm{nM} \text { dexamethasone } \\
50 \mu \mathrm{g} / \mathrm{ml} \text { ascorbic acid } \\
10 \mathrm{mM} \text { sodium } \beta \text {-glyceryl } \\
\text { phosphate }\end{array}$ & $\begin{array}{l}\text { Hyclone } \\
\text { Sigma-Aldrich } \\
\text { Sigma-Aldrich } \\
\text { Sigma-Aldrich }\end{array}$ \\
\hline
\end{tabular}

Table 2 Primer sequences used for $\mathrm{qPCR}$ 


\begin{tabular}{|lll|}
\hline Gene & Primer sequence forward 5'- 3' $^{\prime}$ & Primer sequence reverse 5' 3' $^{\prime}$ \\
\hline Pecam1 & TTGGCACCATGAACAAACTAGCA & CGCTTCGGAGACTGGTCACA \\
\hline CD34 & TGCCGTCTGTCAATGTTTCTGATTA & TCGGATTCCTGAACATTTGATGTC \\
\hline Kdr & AATGCCCATGACCAAGAATGTG & GGATAGAGCCGCGTGTCTGAA \\
\hline Nos 2 & CTCACTGTGGCTGTGGTCACCTA & GGGTCTTCGGGCTTCAGGTTA \\
\hline Nos 3 & GCGGCTGGTACATGAGTTCAGA & AGATCCATGCAGACAGCCACA \\
\hline TIMP-2 & GACACGCTTAGCATCACCCAGA & CTGTGACCCAGTCCATCCAGAG \\
\hline MMP-14 & GAGAACTTCGTGTTGCCTGATGAC & TTCTGGGCTTATCTGGGACAGAG \\
\hline GAPDH & GGACCAGGTTGTCTCCTGTG & CACCTGGAGTACCGGATGT \\
\hline
\end{tabular}

\section{Figures}

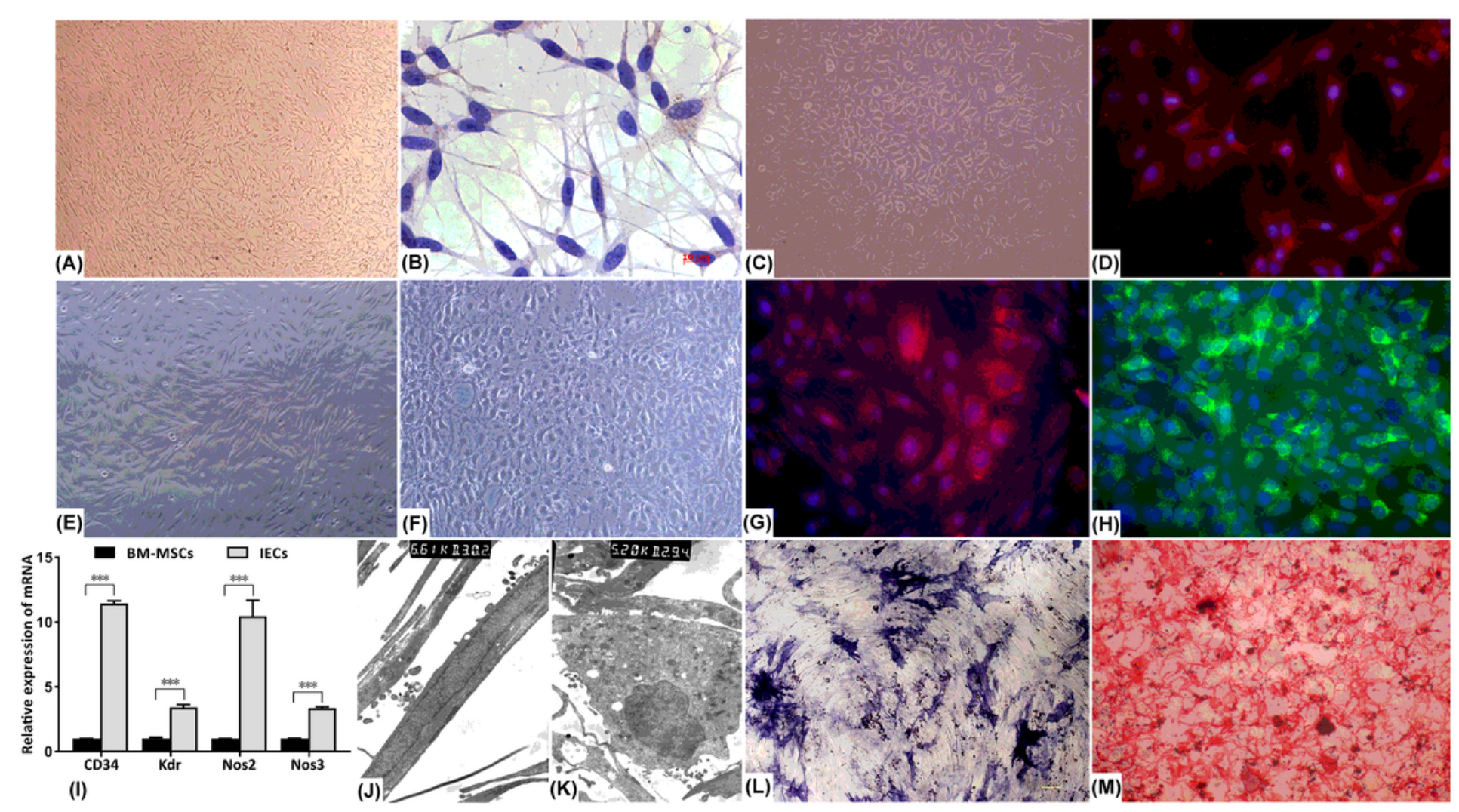

\section{Figure 1}

Cell culture and identification. (A) SCs at passage 2 at the original magnification of $40 \times$ (the same as below). (B) S100 immunocytochemical staining of SCs (400x). (C) AECs at passage 2 (100x). (D) vWF immunofluorescence staining of AECs (400x). (E) BM-MSCs at passage 3 (100x). (F) IECs after induction 
for 21 days (100x). (G) vWF immunofluorescence staining of IECs (400x). (H) Positive Pecam-1 immunofluorescence staining of IECs (400x). (I) CD34, Kdr, Nos2 and Nos3 expression levels in BM-MSCs and IECs, as assessed via real-time qPCR. (J) TEM image of BM-MSCs (6 610x). (K) TEM image of IECs after induction for 21 days (5 200x). (L, M) ALP (L) and alizarin red S (M) staining of IOBs after induction for 21 days $(100 x)$
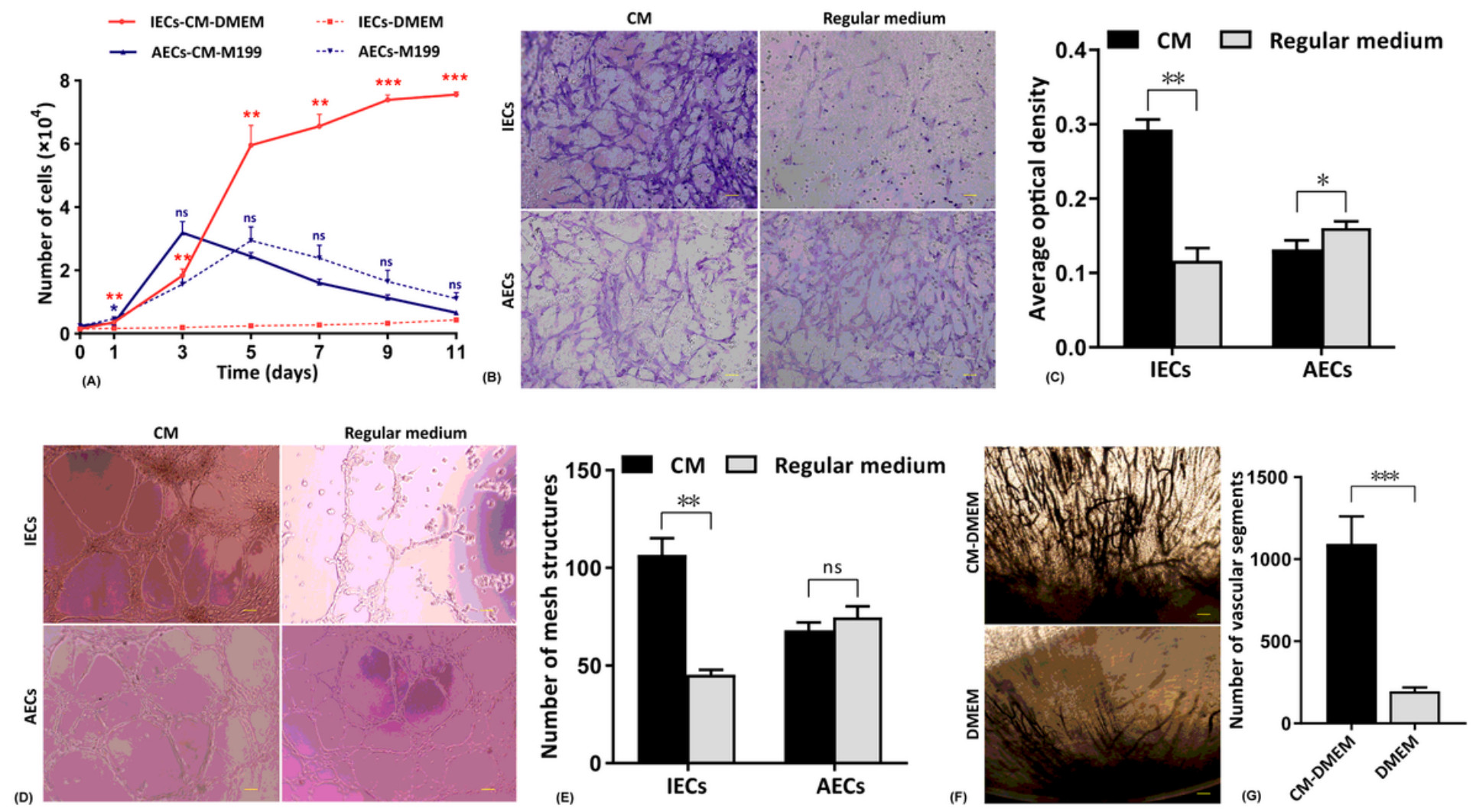

Figure 2

The effects of SC-CM on endothelial cells. (A) Proliferation curves of IECs and AECs in SC-CM and regular medium. (B) Migrated IECs or AECs in the SC-CM wells and regular medium wells (200x). (C) OD values of the eluted fluid of the cell-migrated membranes in each group. (D) Capillary-like tube structures formed by IECs or AECs in Matrigel of SC-CM wells and regular medium wells (100x). (E) Capillary-like mesh structure counting of each group. (F) New vessels perfused by ink in the rat corneas of each group (100x). (G) Vascular segment counting of the rat corneas of each group 

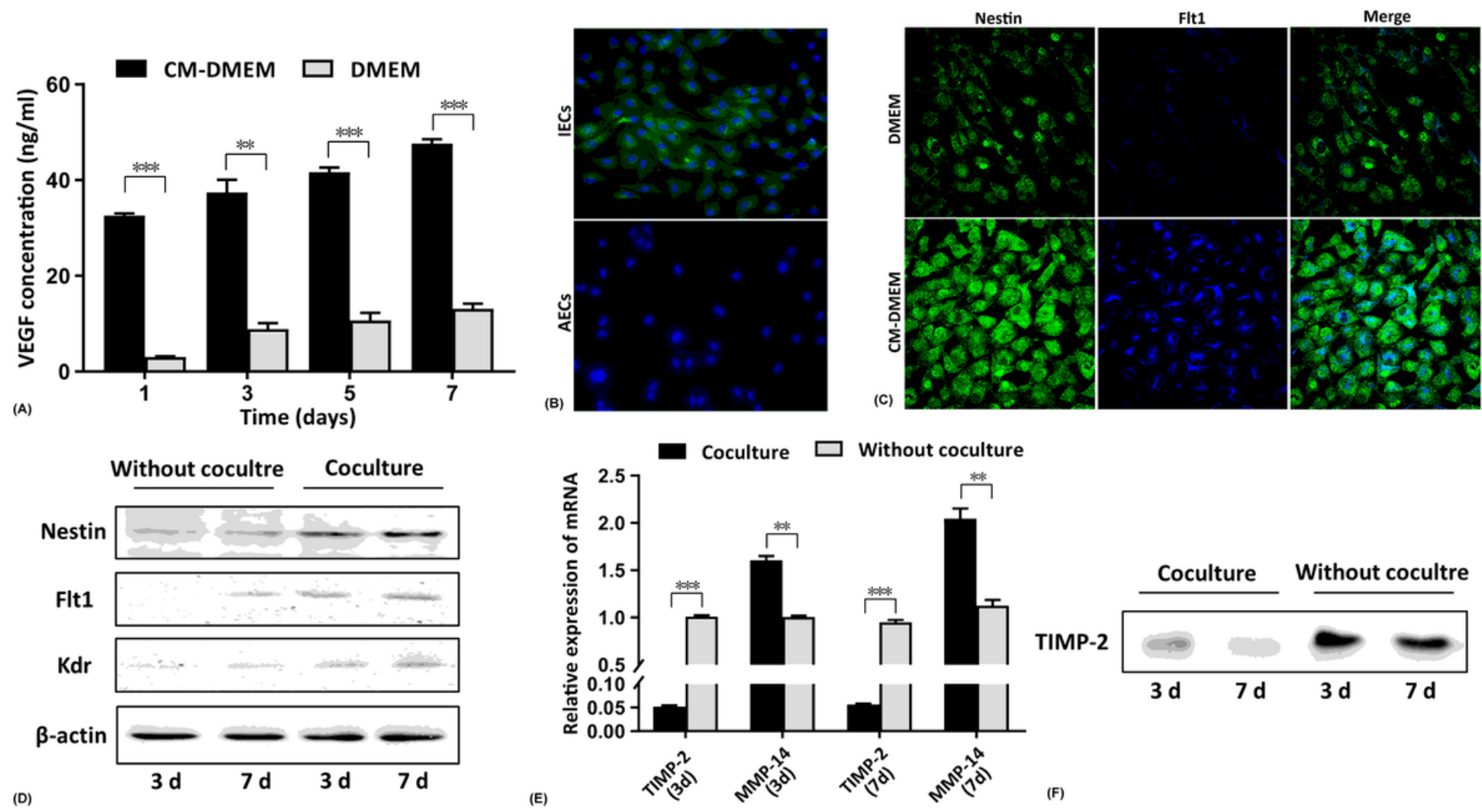

(D)

\section{Figure 3}

The interaction of SCs and endothelial cells. (A) VEGF secretion levels in the supernatants in each group as determined by ELISA. (B) Nestin expression in IECs and AECs by immunofluorescence staining (400x). (C) Nestin and Flt1 expression in IECs in SC-CM-DMEM or DMEM by double immunofluorescence staining. (D) Nestin, Flt1 and Kdr expression levels in IECs with or without coculture at days 3 and 7 by WB. (E) TIMP-2 and MMP-14 expression levels in SCs cocultured with IECs and without coculture by qPCR. (F) Secreted TIMP-2 expression in the supernatants of the coculture system and the control wells 

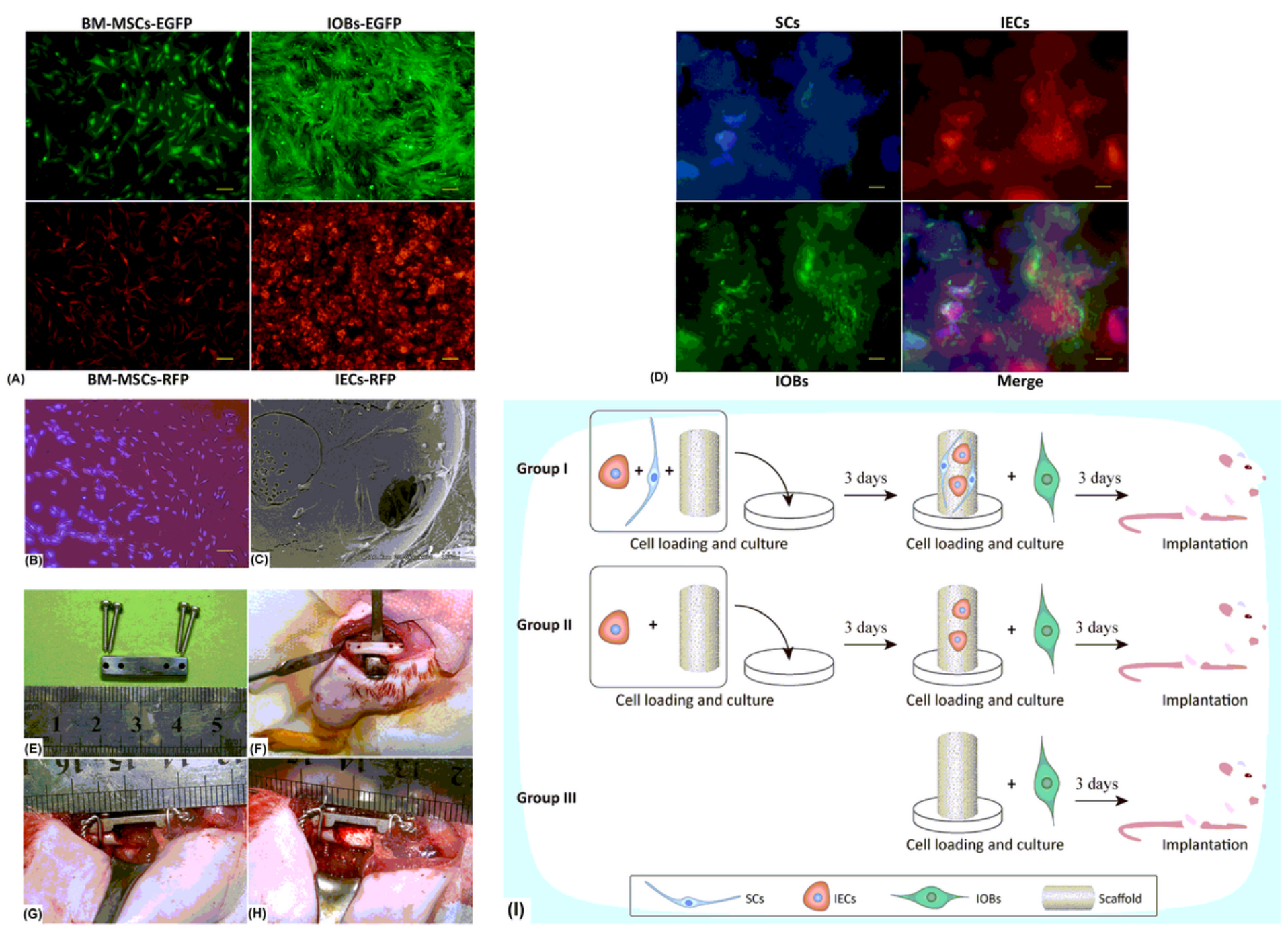

\section{Figure 4}

Prevascularized scaffolds loaded with labeled cells were applied in rat models. (A) BM-MSCs, IOBs expressing EGFP and IECs expressing RFP (100x). (B) SCs labeled with Hoechst 33342 (100x). (C) SCs, IECs and IOBs adhered and grew on $\beta$-TCP scaffolds observed by SEM. (D) labeled SCs, IOBs-EGFP and IECs-RFP grew on the $\beta$-TCP scaffolds to make prevascularized TEBGs (100x). (E) The internal fixation with steel plate applied in rat operations. (F) Femur exposed and drilled for internal fixation. (G) 6-mmlong defect of the middle femur and the steel plate implanted for internal fixation. $(\mathrm{H})$ Prevascularized TEBG implanted into the femur defect. (I) The design of the time schedule and group setting during the experiment 

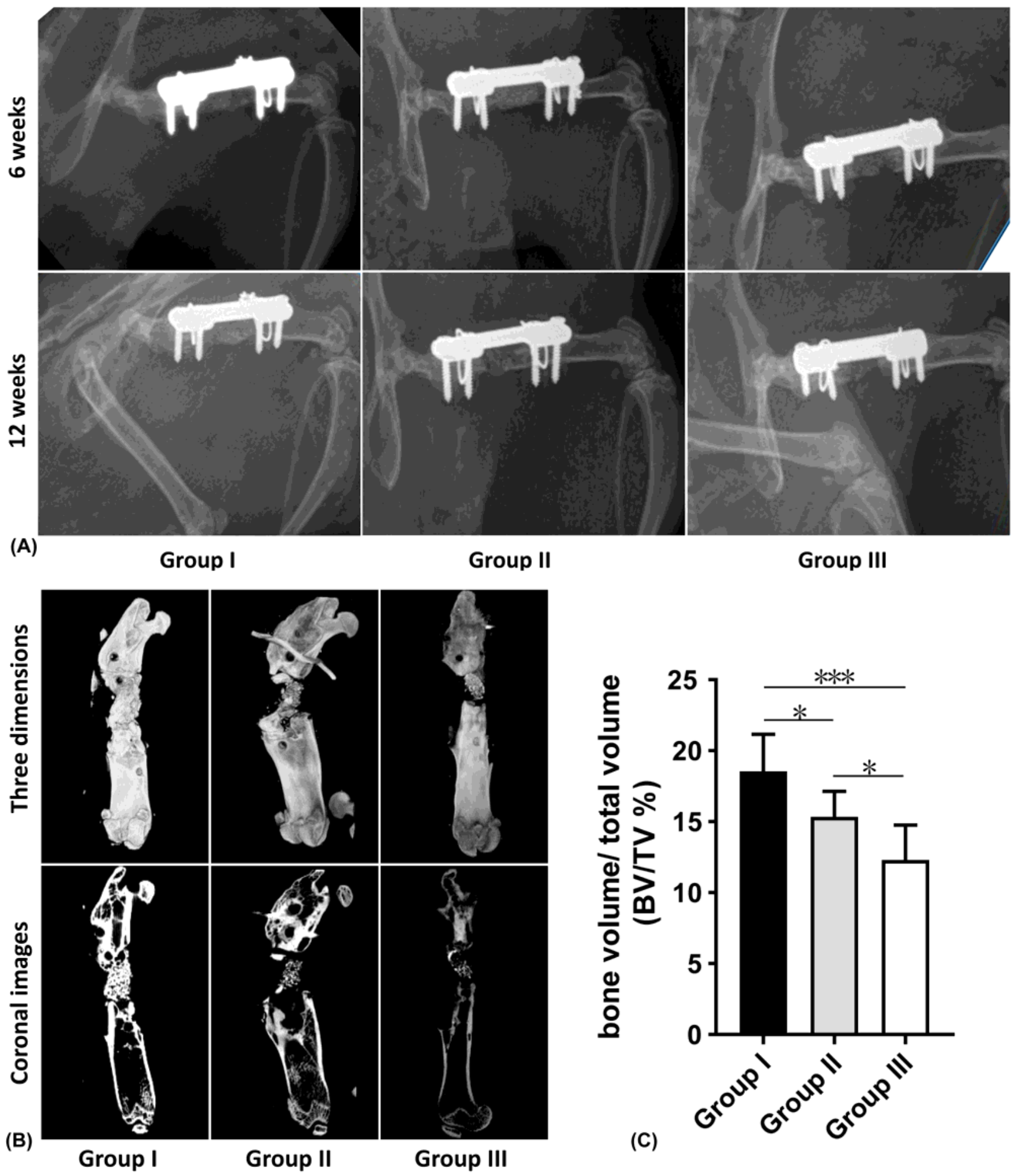

Figure 5

Radiological assessment of TEBGs in vivo. (A) X-ray images after operation 6 weeks and 12 weeks of three groups. (B-C) The micro-CT images of three groups (B) and the comparison of BV/TV \% values (C) after removing the internal fixations at 12 weeks postoperatively 


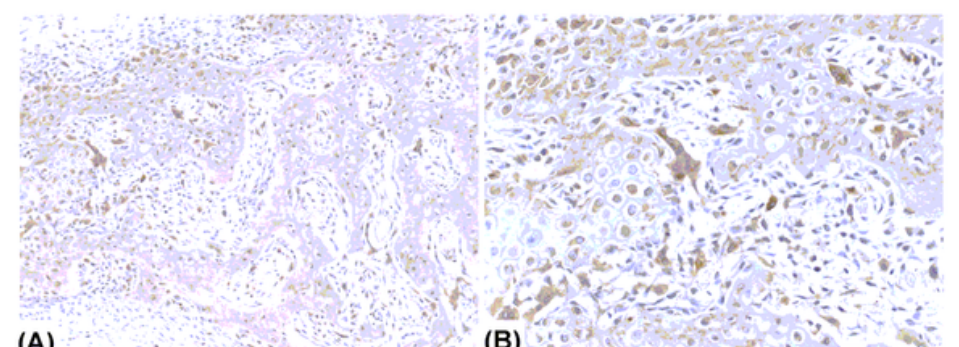

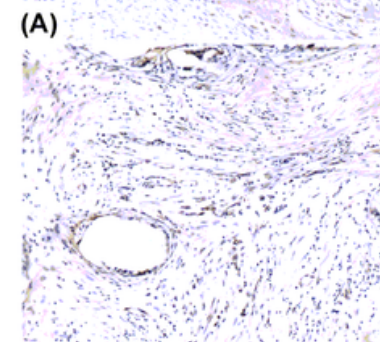

(C)

(E)

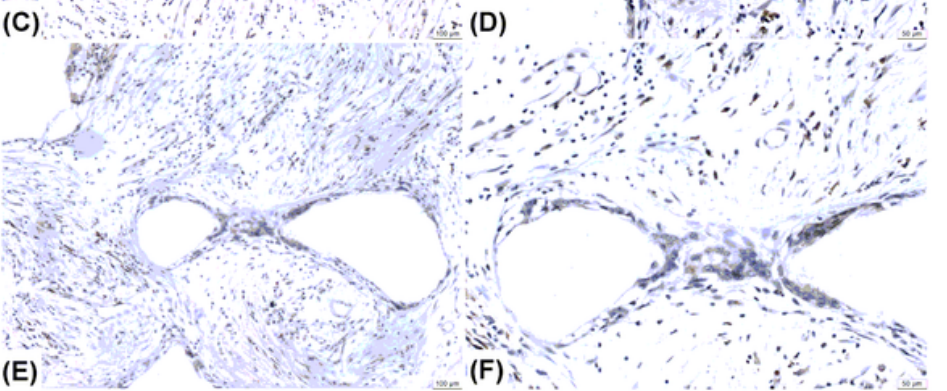

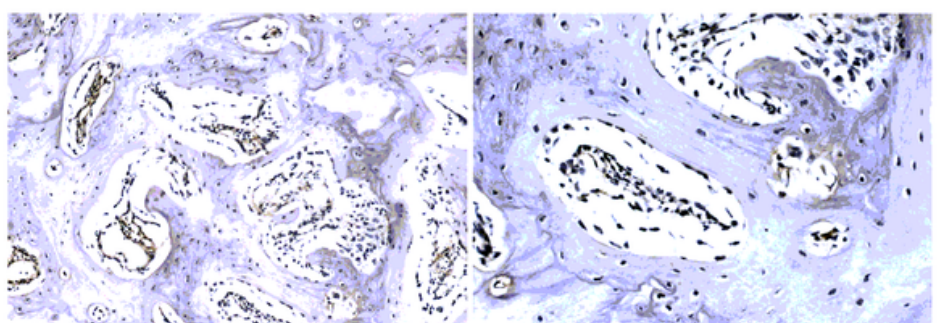

(G)

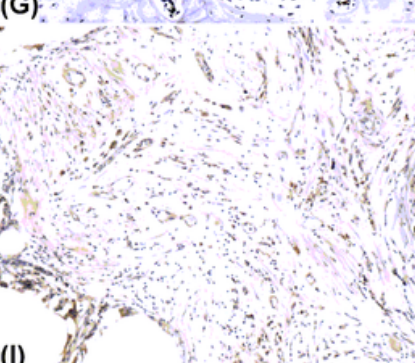

$-(\mathrm{H})$

$\rightarrow(H)$
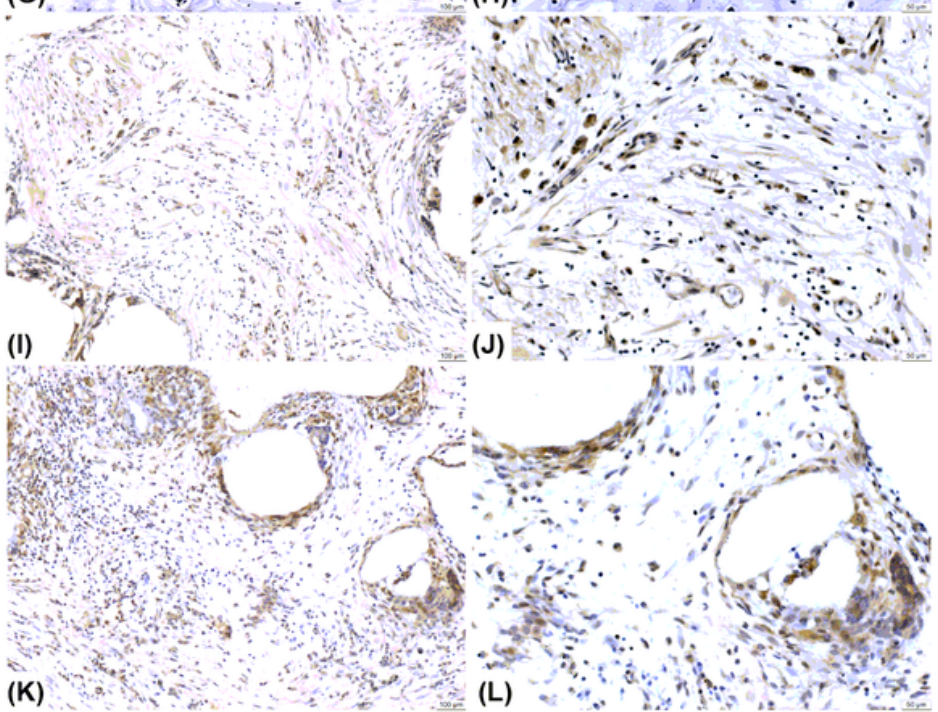
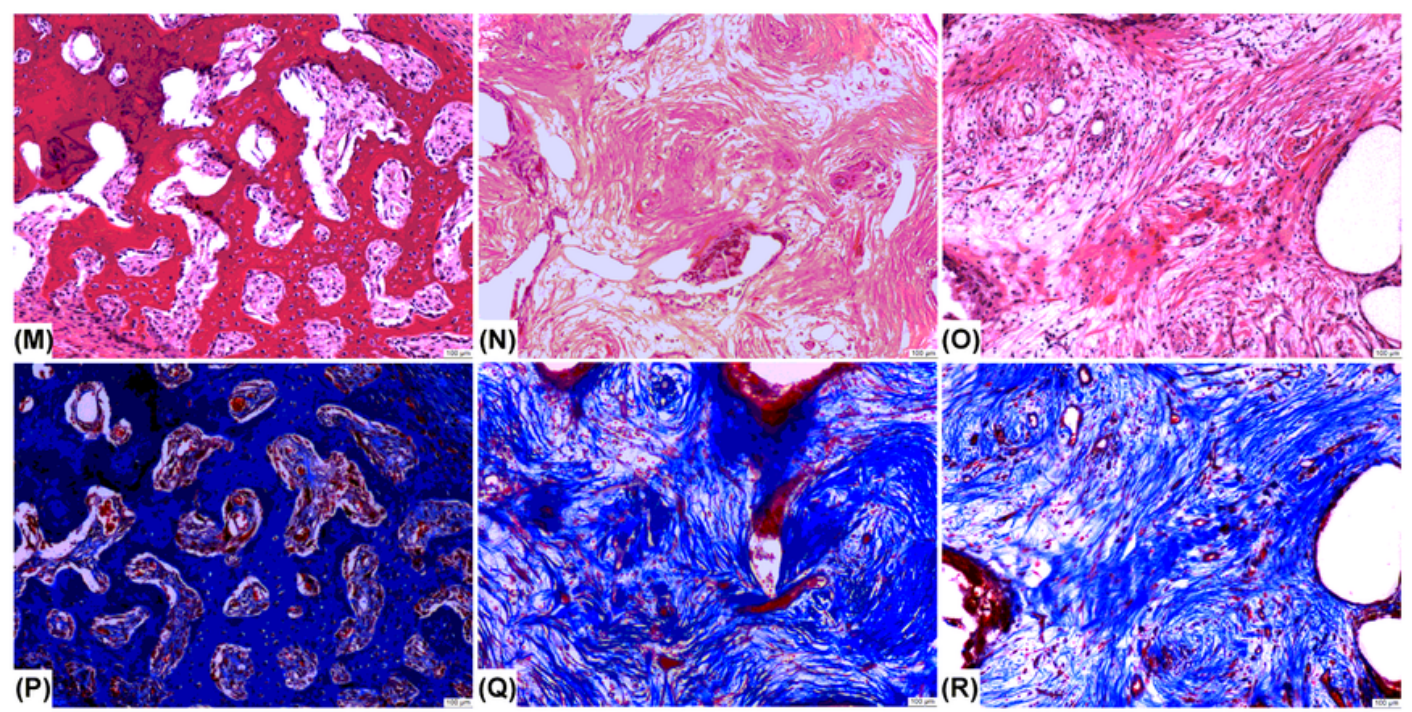

\section{Figure 6}

Histological assessment of TEBGs in vivo. (A-F) IHC staining of nestin at 12 weeks in groups I (A, B), II (C, D) and III (E, F). (G-L) IHC staining of TIMP-2 at 12 weeks in groups I $(G, H)$, II $(I, J)$ and III $(K, L)$. (M-R), H\&E and Masson staining at 12 weeks in groups I $(M, P), I I(N, Q)$ and III $(O, R)$ 

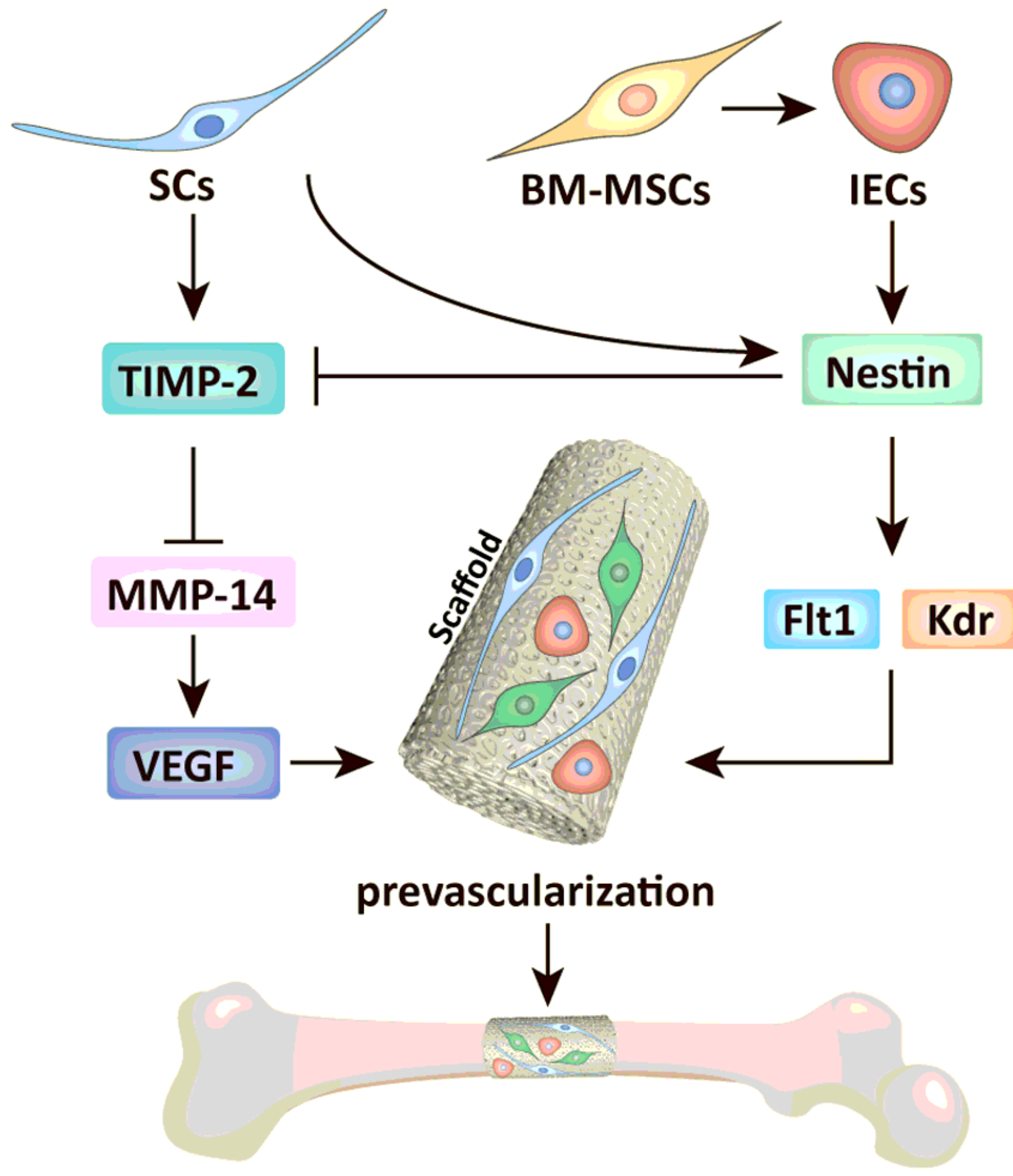

Figure 7

A schematic diagram of the interactions between SCs and endothelial cells in TEBG. SCs promoted nestin and VEGF receptor expression of BM-MSC derived IECs, and IECs inhibited TIMP-2 secretion of SCs by autologous nestin expression to promote activity of MMP-14 and VEGF, eventually efficiently induced prevascularization and osteogenesis of TEBG 